\title{
Demonstration of Structural Alterations in Experimental Corneal Infectious Model Using Multiphoton Microscopy
}

\author{
Wen Lo ${ }^{1}$, Hsin-Yuan Tan ${ }^{2,3 \#}$, Yuh-Ling Chang ${ }^{1}$, Yen Sun ${ }^{1}$, Sung-Jan Lin ${ }^{4}$, Shiou-Hwa Jee ${ }^{4}$, Chen- \\ Yuan Dong ${ }^{1 *}$ \\ ${ }^{1}$ Department of Physics, National Taiwan University, Taipei 106, Taiwan \\ ${ }^{2}$ Institute of Medical Engineering, College of Medicine and College of Engineering, Taipei 100, \\ Taiwan \\ ${ }^{3}$ Department of Ophthalmology, College of Medicine, Chang Gung University and Chang Gung \\ Memorial Hospital, Linko 333, Taiwan \\ ${ }^{4}$ Department of Dermatology, College of Medicine, National Taiwan University and National \\ Taiwan University Hospital, Taipei 100, Taiwan
}

\begin{abstract}
The aim of this study is to assess the application of multiphoton autofluorescence and second harmonic generation (SHG) microscopy for investigating the structural alterations and the pattern of microbial spreading during corneal infectious process in an in vitro organ culture model. The autofluorescence spectrum derived from pathogens allows us to monitoring the pattern of microbial spreading within corneal lamellae. In addition, the destruction and regeneration of second harmonic generating collagen during infectious process can also be monitored in a non-invasive fashion. Therefore we propose that multiphoton microscopy may potentially be applied as an effective monitoring tool for corneal infection studies.
\end{abstract}

Keywords: multiphoton autofluorescence, second harmonic generation, cornea, infection, organ culture.

Address correspondences to: ${ }^{*} \mathrm{H}$. Y. Tan at d93548010@ntu.edu.tw and ${ }^{*} \mathrm{C}$. Y. Dong at cydong@phys.ntu.edu.tw

\section{INTRODUCTION}

Cornea infection is a common cause of severe vision impairment. Infectious keratitis patients need intensively and immediately medical attention. Although infectious keratitis is a global eye disease, regional differences in pathogens do exist. Fast and accurate diagnosis can help doctors to choose proper antimicrobial treatment in time. Currently, the clinical diagnosis still relies on the laboratory culture system which may need several days to process. In the case of fungal infection, the diagnostic procedure usually involves more than one week in vitro culturing for pathogen identification. Therefore, an in vivo imaging technique which can provide pathological information in the early stage of infectious keratitis is helpful for clinical diagnosis and improved therapy.

Although confocal microscopy has been applied to the pathological imaging of infectious keratitis(1-4), there remain limitations to its application. The turbidity of infected cornea reduces the imaging depth of confocal microscopy and the absence of extra cellular matrix information also restricted its application. In recent years, multiphoton microscopy has been wildly applied to a variety of biomedical problems due to its reduced photodamage and prolonged penetration depth(5-8). The minimal invasion and deep penetration associated with multiphoton microscopy also render this technique a useful in vivo imaging method in clinical examination. In multiphoton imaging, cytoplasmic autofluorescence provides the morphological and spectral information of cells, pathogens and other tissue components. Moreover, second harmonic generation (SHG) from collagen provides visualization of structural changes of extra cellular matrix (9-11).

Ophthalmic Technologies XVII, edited by Fabrice Manns, Per G. Soederberg, Arthur Ho, Bruce E. Stuck, Michael Belkin, Proc. of SPIE Vol. 6426, 64260Y, (2007) · 1605-7422/07/\$18 · doi: 10.1117/12.699468 
In this work, bovine corneas are excised and cultured in Petri dish and a long term imaging chamber. This approach allows us to dynamically investigate the infection process.

\section{MATERIALS AND METHODS}

\subsection{Mutiphoton microscope}

The multiphoton microscope (Fig. 1) we used in this study is a homebuilt system based on a commercial upright microscope (Nikon, E800, Japan.) In order to obtain images at both high resolution and large scale features, a motorized translational stage (H101, Prior, UK) is adapted to the upright microscope. A titanium-sapphire pulse laser (Tsunami, Spectra Physics, Mountain View, CA) operating at the repetition rate of $80 \mathrm{MHz}$ and the wavelength of $780 \mathrm{~nm}$ was used for excitation. A water immersion objective (SFluor, WI, 40X, N.A. 0.8, Nikon) is used to focus laser and acquire multiphoton excited autofluorescence (AF) and second harmonic generation (SHG) from the cornea specimens. To eliminate the reflection from sample, the obtained signals first pass through a short-pass dichroic mirror (700dcspruv3p, Chroma Technology, Rockingham, VT). Prior to reaching the detectors, the broadband autofluorescence and SHG are separated by a secondary dichroic mirror ( $435 \mathrm{dcxr}$, Chroma Technology) and filtered by two band-pass filters respectively (AF: E435lp-700sp, SHG: HQ390/20, Chroma Technology). Photon-counting photomultiplier tubes (R7400P, Hamamatsu, Japan) were used to detect the AF and SHG signals.

\subsection{Long term imaging chamber}

To obtain dynamical information of the infection processes, a corneal culture chamber (Fig. 2) was designed for long term and repeated imaging. The chamber is made of biocompatible polycarbonate (LEXAN TM, General Electronic Company, Fairfield, CT). In our design, the bovine corneal-sclera button is placed in the primary chamber which is concealed by a height adjustable cover with an observing window. The observing window is sealed with a $0.17 \mathrm{~mm}$ thick cover glass for obtaining highly resulted mulitphoton images. The secondary chamber adjacent to the primary chamber has a cap with sterile air filter which allows gas exchange. All the parts are conjoined by biocompatible adhesive (461, Loctite Corporation, Hartford, CT).

\subsection{Bovine corneal model}

Intact bovine eye bulbs are obtained from a local market. To avoid unwanted infection, the eyes were first disinfected with $10 \%$ povidone iodine then rinsed by sterilized phosphate-buffered saline (PBS). In the first experiment, central corneal buttons are later excised by a $7.5 \mathrm{~mm}$ corneal trephine and cultured in Petri dishes for one day prior to the infection experiment.

In the long term observation experiment, corneal-sclera buttons are excised and placed with the epithelium faced down in a sterile container containing PBS. The endothelial corneal cavity was then filled with $1 \%$ agarose gel dissolved in PBS at $40{ }^{\circ} \mathrm{C}$. The cornea along with its supporting gel was later inverted and transferred to the long term imaging chamber. Approximate $4 \mathrm{ml}$ of DMEM was added drop-wise to the central cornea until the limbal conjunctiva was covered and with the epithelial surface exposed to the air. The long term imaging chamber was later removed to an incubator with $5 \% \mathrm{CO}_{2}$ at $37^{\circ} \mathrm{C}$.

\subsection{Pseudomonas aeruginosa infection induction}

$10 \mu \mathrm{l}$ of pathogen suspension (Pseudomonas aeruginosa, $10^{6} \sim 10^{7}$ organism $/ \mathrm{ml}$ ) was injected into each cornea button by 30 gauge needles. In the Petri dish experiment, the infected corneal buttons were cultured in incubator for several time intervals $(0,6,12,24$ hours) then directly mounted on microscope for imaging. In the control group of this experiment, the same volume of PBS was injected in to corneal buttons.

In the continuous observation experiment, after injection of the pathogen suspension, the corneal-sclera button was sealed in the long term imaging chamber. During the imaging process, the chamber was directly mounted on the microscope under a temperature controlled environment $\left(37^{\circ} \mathrm{C}\right)$. 


\section{RESULTS}

In both the Petri-dish and chamber cultured experiments, the injected pathogens can be visualized within the corneal stroma by detecting autofluorescence detection. In Fig. 3, the multiphoton image of isolated pathogen from colony shows the morphological characters of Pseudomonas aeruginosa. In, Fig. 4 and 5, the SHG images provide the information collagen orientation in corneal stroma. With the aid of visualizing collagen fibers, we are able to investigate the interaction between pathogens and extra cellular matrix.

In Fig. 4 (infected corneas), there is an obvious trend that autofluorescent area increased with the infecting time while the SHG area decreased. The autofluorescence images of 0 and 6 hours in Fig. 4 shows the injected pathogen spread along the inter-fibril space in the corneal stroma. As the infection time increased, the SHG area shrunk due to the destruction of collagen stroma while the autofluorescence area enlarged. This result suggests that the increased autofluorescence may be due to both pathogenesis and tissue response. The same trend, however, was not observed from the cornea specimens in the control group (Fig. 5).

For further investigation, the autofluorescence-SHG index (ASI) was introduced to quantitatively analyze this result. The definition of ASI is the size difference between the autofluorescence and SHG areas in an image divided by their sum $([\mathrm{AF}-\mathrm{SHG}] /[\mathrm{AF}+\mathrm{SHG}])(12)$. The results of the ASI analysis are shown in Fig. 6. In the infection group, the ASI is about -1 at the start of infection then increases with time until 12 hours after injecting the pathogen suspension in corneal stroma. However, the ASI remains -1 in control group at every observing time point $(0,6,12,24$ hours). This result suggests that the ASI can be used to quantify the progression of corneal infection and the in vitro Pseudomonas aeruginosa infection is complete in 12 hours.

In the long term imaging experiment, multiphoton image of the same sample can be repeatedly acquired by applying our imaging chamber. Fig. 7 demonstrates that two closed spaced regions in the infected sample can be visualized after three hours culture. The ability of acquiring high resolution image in the same location at different time point provides the feasibility of dynamically investigating the infection mechanism. Fig. 7 demonstrates the pathogen spreading in the inter-fibril space in corneal stroma. After 3 hours spreading, the pathogens are still attached to the collagen fibrils. This result suggests that the proliferation and migration of Pseudomonas aeruginosa in corneal stroma is related to the interaction with extra cellular matrix.

\section{CONCLUSIONS}

Multiphoton autofluorescence and second harmonic generation microscopy can be used to identify the presence of pathogens and the structural alterations within corneal infectious models. The results of our Petri dish cultured experiment show the increased autofluorescence and decreased SHG in Pseudomonas infected corneas. We also quantitatively analyze this result and introduced an index (ASI) to describe the changes that occurred within the corneal tissues. The ASI successfully describes the progression of infection and shows that in vitro Pseudomonas aeruginosa infection is complete within 12 hours.

In long term imaging experiment, our results suggest that our long term imaging chamber is very useful for the dynamic and mechanistic investigation of corneal infection. In addition, our results also suggest that the pathogen/ extra cellular matrix interaction may be critical to the proliferation and migration of bacterial pathogens. 


\section{FIGURES}

\section{The Setup of Multiphoton Microscope}

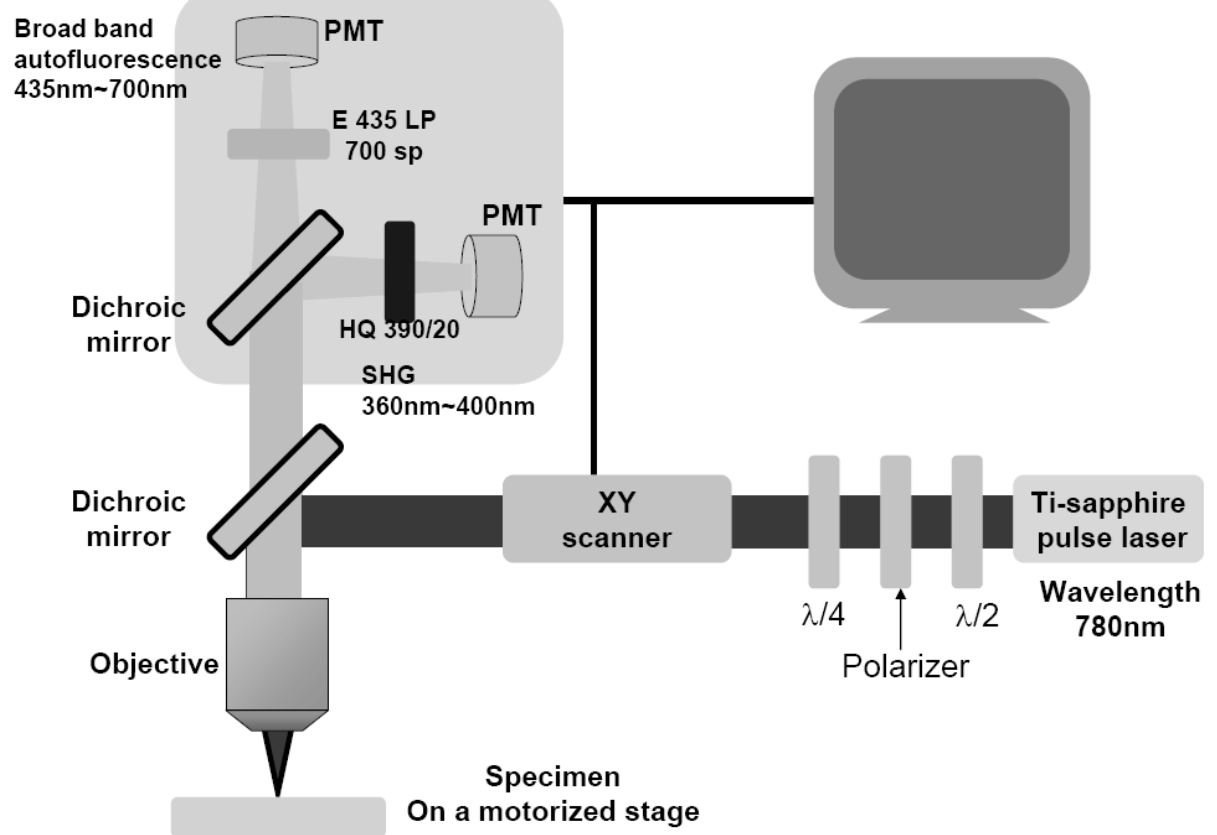

Fig. 1 The schematic setup of our homebuilt multiphoton microscope.

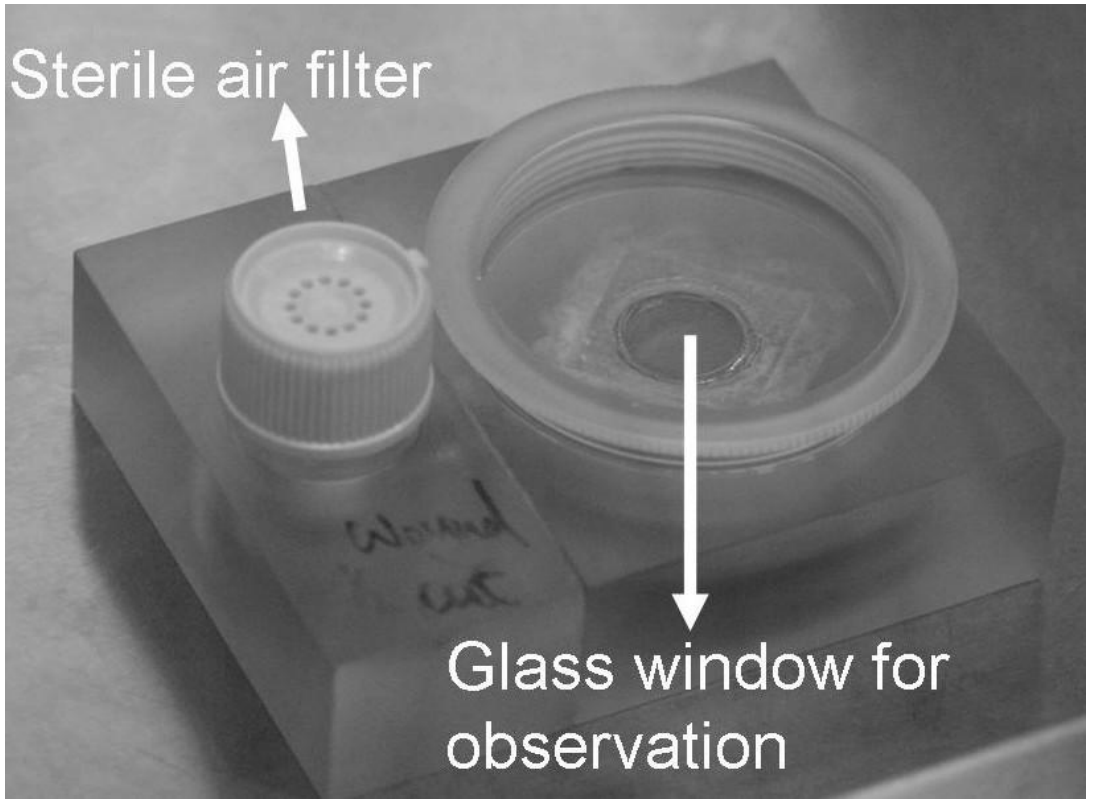

Fig. 2 The in vitro long term imaging chamber made of biocompatible and autoclavable polycarbonate. The chamber can be directly adapted to an upright microscope for high resolution imaging. 


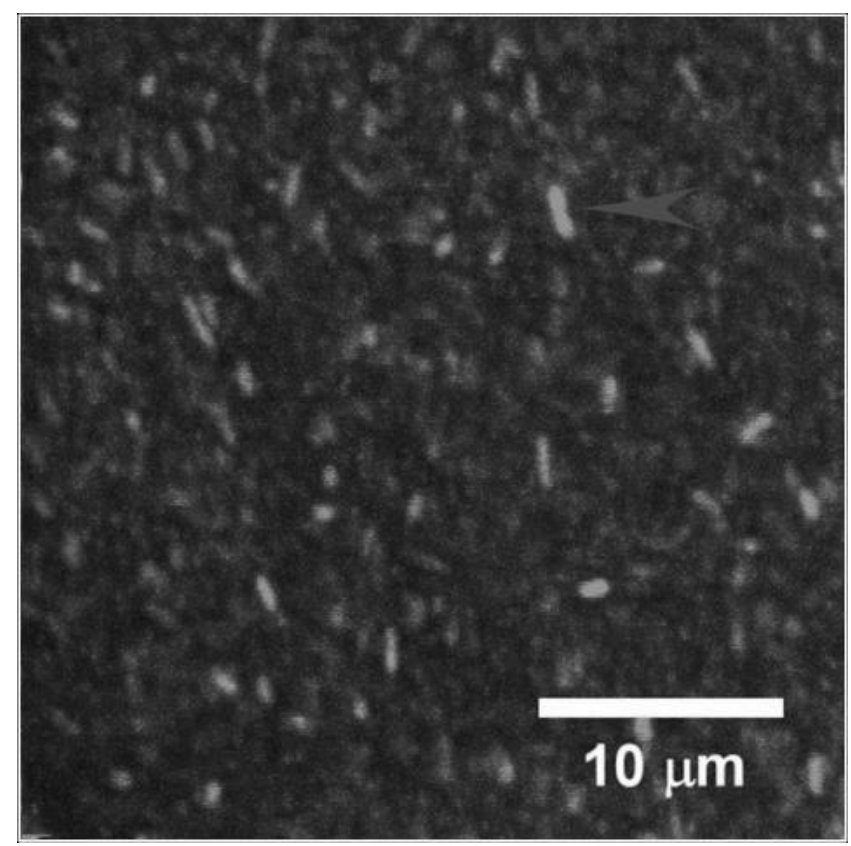

Fig. 3 The multiphoton image of isolated Pseudomonas aeruginosa form colony. The multiphoton excited autofluorescence provide the visualization of the bacterial pathogen which shows the rod-shaped Pseudomonas aeruginosa.

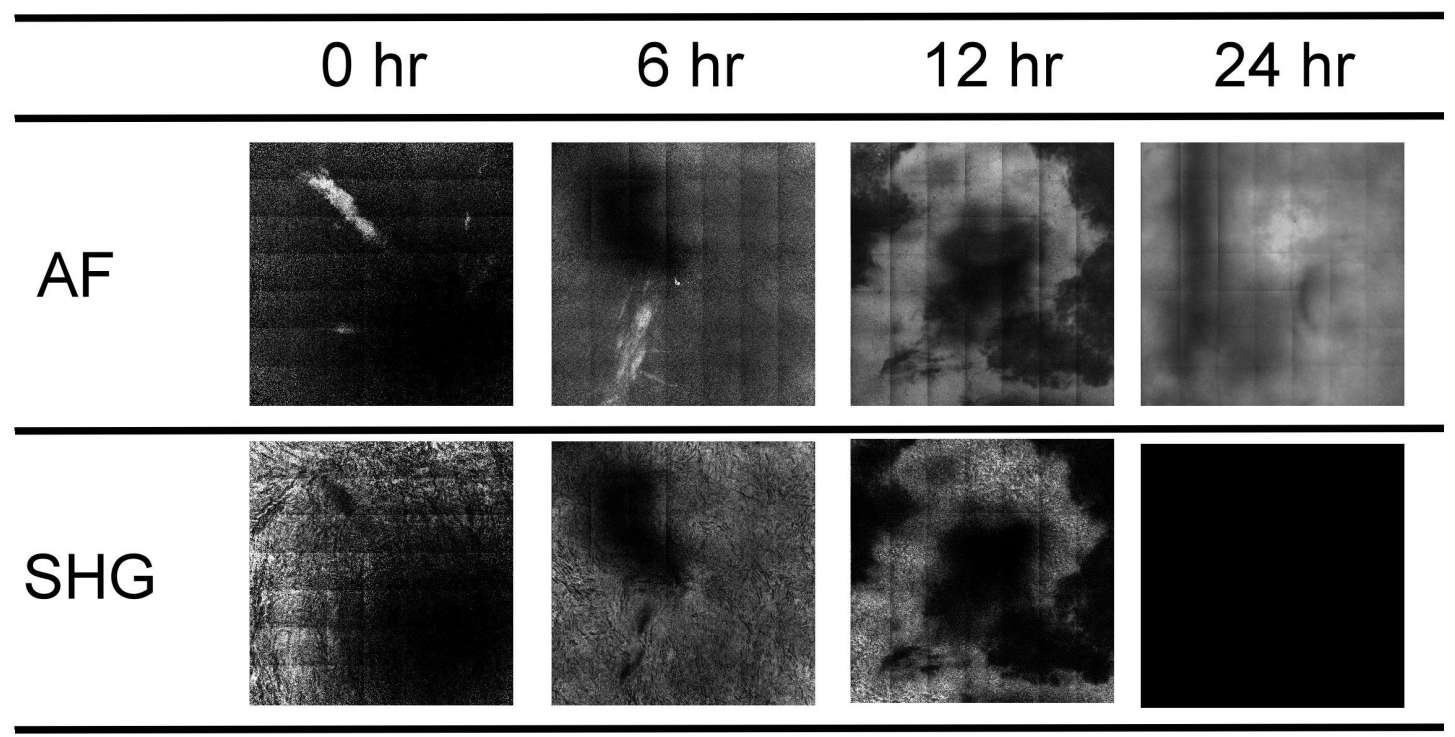

Fig. 4 Multiphoton images of corneal buttons at different infection grades. After injecting pathogen suspension, the bovine corneal button has been cultured in an incubator for $0,6,12,24$ hours prior to multiphoton imaging. As the infection time increased, the autofluorescence area increased where as the SHG area decreased. (Image size: $1.3 \mathrm{~mm}$ by 1.3 $\mathrm{mm})$ 


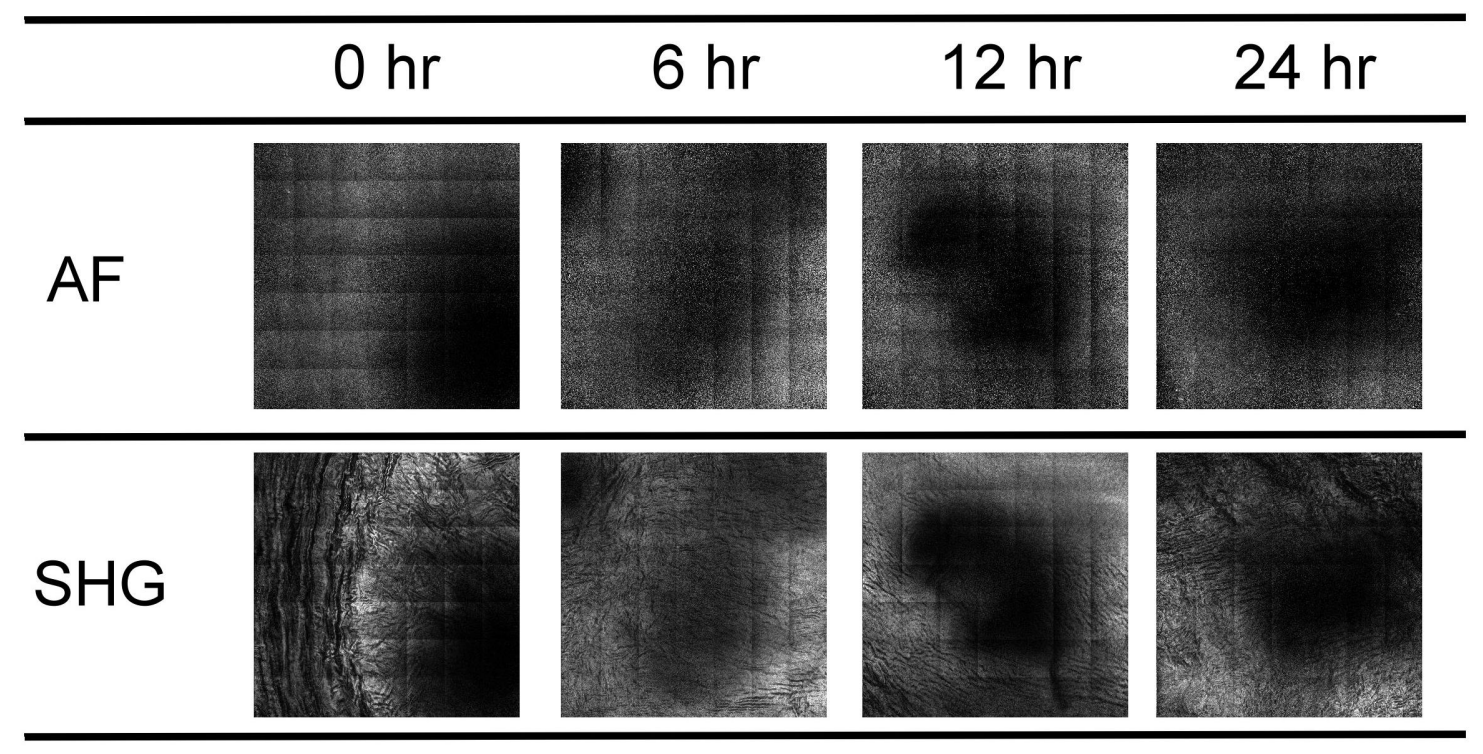

Fig. 5 Multiphoton images of corneal button in the control group. The control group corneal buttons were injected with PBS then cultured in Petri dish for 0, 6, 12, 24 hours. These images show that there is no significant variation of morphology, autofluorescence and SHG. (Image size $1.3 \mathrm{~mm}$ by $1.3 \mathrm{~mm}$ ).

- Control ASI \ Infection ASI

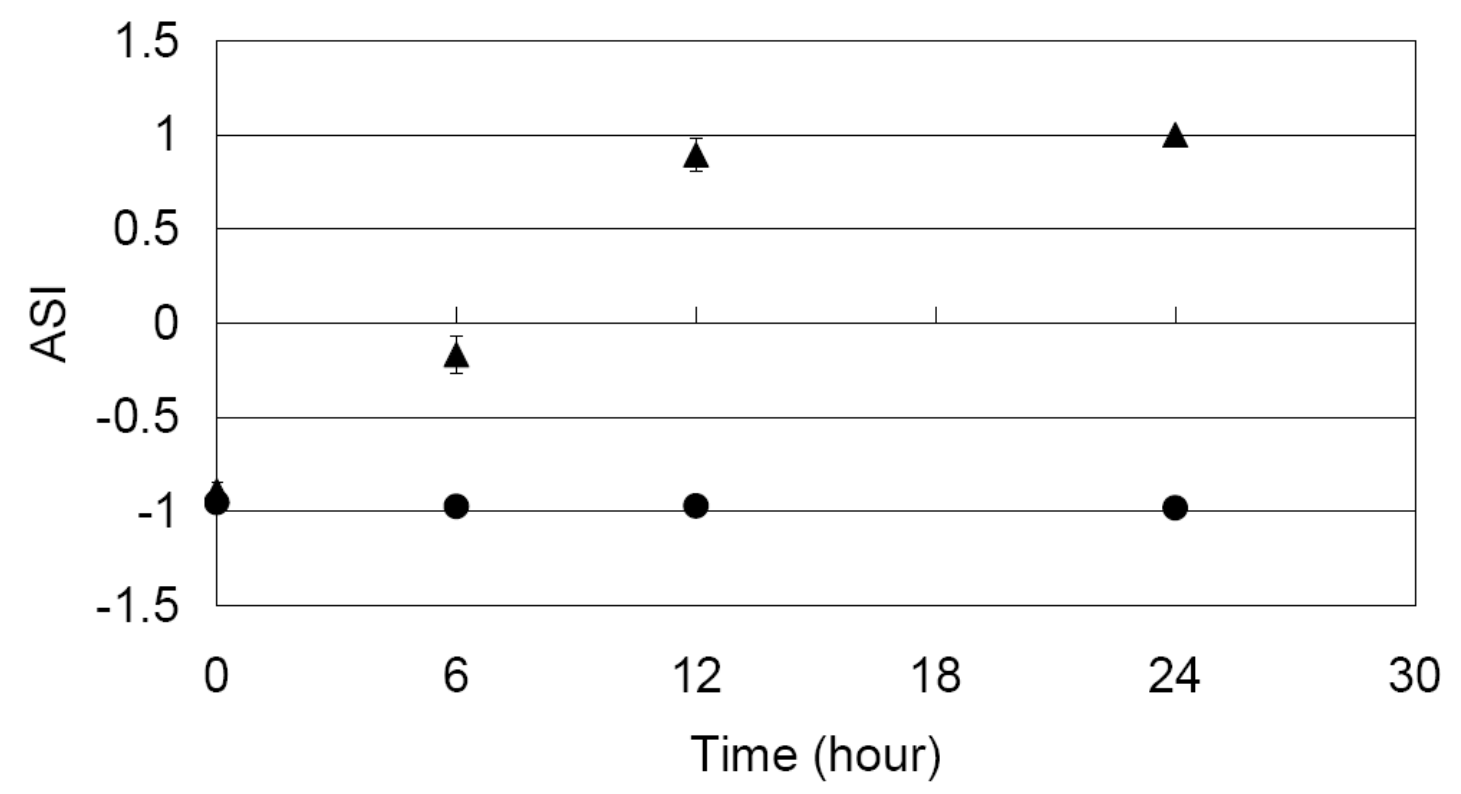

Fig. 6 Quantitative analysis of Pseudomonas aeruginosa infection by ASI. 


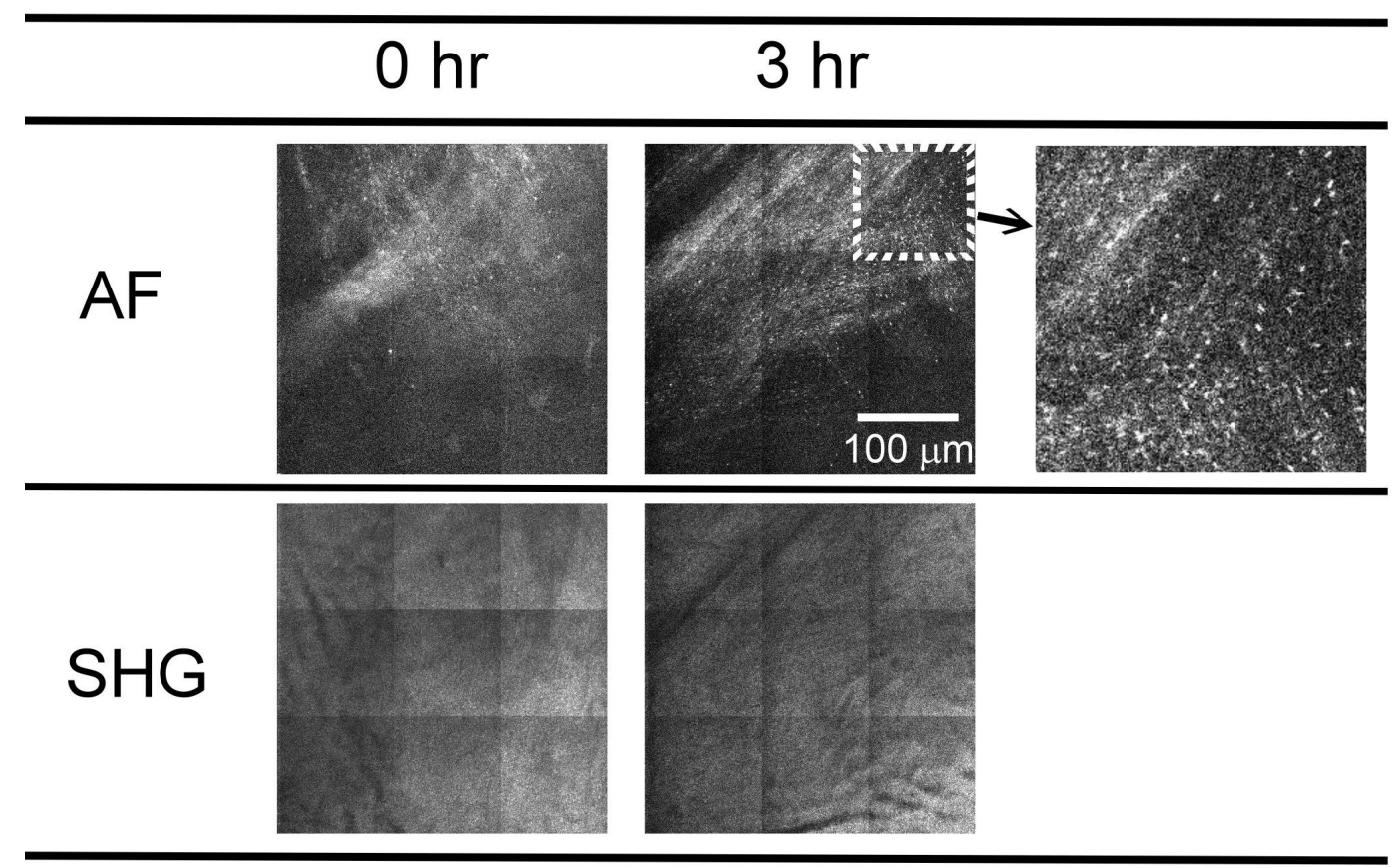

Fig. 7 Multiphoton images of infected corneal-sclera button obtained sequentially.

\section{ACKNOWLEDGMENT}

We like to acknowledge the support of the National Science Council, Taiwan and the National Research Program for Genomic Medicine (NRPGM), Taiwan (NSC95-3112-B-002-018). This project was completed using the Optical Molecular Imaging Microscopy Core Facility (A5) of NRPGM.

\section{REFERENCES:}

1. I. Jalbert, F. Stapleton, E. Papas, D. F. Sweeney and M. Coroneo, "In vivo confocal microscopy of the human cornea," British Journal of Ophthalmology 87(2), 225-236 (2003)

2. W. D. Mathers, S. E. Nelson, J. L. Lane, M. E. Wilson, R. C. Allen and R. Folberg, "Confirmation of confocal microscopy diagnosis of Acanthamoeba keratitis using polymerase chain reaction analysis," Archives of Ophthalmology 118(2), 178-183 (2000)

3. D. R. Pfister, J. D. Cameron, J. H. Krachmer and E. J. Holland, "Confocal microscopy findings of Acanthamoeba keratitis," American Journal of Ophthalmology 121(2), 119-128 (1996)

4. J. E. Sutphin, A. L. Kantor, W. D. Mathers and M. G. Mehaffey, "Evaluation of infectious crystalline keratitis with confocal microscopy in a case series," Cornea 16(1), 21-26 (1997)

5. J. M. Squirrell, D. L. Wokosin, J. G. White and B. D. Bavister, "Long-term two-photon fluorescence imaging of mammalian embryos without compromising viability," Nature Biotechnology 17(8), 763-767 (1999)

6. W. Denk, J. H. Strickler and W. W. Webb, "2-Photon Laser Scanning Fluorescence Microscopy," Science 248(4951), 73-76 (1990)

7. P. T. C. So, C. Y. Dong, B. R. Masters and K. M. Berland, "Two-photon excitation fluorescence microscopy," Annual Review of Biomedical Engineering 2(399-429 (2000) 
8. M. J. Miller, S. H. Wei, I. Parker and M. D. Cahalan, "Two-photon imaging of lymphocyte motility and antigen response in intact lymph node," Science 296(5574), 1869-1873 (2002)

9. H. Y. Tan, Y. Sun, W. Lo, S. J. Lin, C. H. Hsiao, J. F. Chen, S. C. M. Huang, W. C. Lin, S. H. Jee, H. S. Yu and C. Y. Dong, "Multiphoton Fluorescence and Second Harmonic Generation Imaging of the Structural Alterations in Keratoconus Ex Vivo.," Investigative Ophthalmology \& Visual Science (2006)

10. W. Lo, S. W. Teng, H. Y. Tan, K. H. Kim, H. C. Chen, H. S. Lee, Y. F. Chen, P. T. C. So and C. Y. Dong, "Intact Corneal Stroma Visualization of GFP Mouse Revealed by Multiphoton Imaging," Microscopy Research and Technique 69(12), (2006)

11. W. R. Zipfel, R. M. Williams and W. W. Webb, "Nonlinear magic: multiphoton microscopy in the biosciences," Nature Biotechnology 21(11), 1368-1376 (2003)

12. S. Lin, T. Wu, T. Young, W. Lo, S. Jee and C. Dong, "Quantitative determination of photoaging by use of multiphoton fluorescence and second harmonic generation microscopy," Journal of Investigative Dermatology 125(3), A58-a58 (2005) 